\title{
Quantitative and Qualitative Gradient of Pain Experience, Sleep Quality and Psychological Distress in Patients with Different Phenotypes of Temporomandibular Disorders
}

\author{
Hee Hun Choi ${ }^{1}$, Hye-Kyoung Kim ${ }^{2}$, Mee-Eun Kim ${ }^{2}$ \\ ${ }^{1}$ Ipyeonhan Dental Clinic, Suwon, Korea \\ ${ }^{2}$ Department of Oral Medicine, Collage of Dentistry, Dankook University, Cheonan, Korea
}

Received August 18, 2020

Revised September 3, 2020

Accepted September 3, 2020
Correspondence to:

Mee-Eun Kim

Department of Oral Medicine, Collage of Dentistry, Dankook University, 119 Dandaero, Dongnam-gu, Cheonan 31116, Korea Tel: +82-41-550-1915

Fax: +82-505-434-7951

E-mail:meunkim@dankook.ac.kr

https://orcid.org/0000-0001-9332-532X
Purpose: Temporomandibular disorders (TMD) is a mosaic of clinical signs and symptoms that can be regarded as a set of phenotypes that are affected by various factors including pain sensitivity, pain disability, sleep and psychological functioning. The aims of this study were to evaluate association of pain experience, sleep quality and psychological distress with different phenotypes of TMD patients.

Methods: This retrospective study included a cohort $(n=1,858 ; 63.8 \%$ for female, mean age $=34.9 \pm 15.9$ years) of patients with TMD. A set of self-administered questionnaires concerning pain interference (Brief Pain Inventory), pain disability (Graded Chronic Pain Scale), sleep quality (Pittsburg Sleep Questionnaire Index), psychological distress (Symptom Checklist-90 revised), and pain catastrophizing (Pain Catastrophizing Scale) were administered to all participants at the first consultation. All TMD patients were classified into four groups including TMD with internal derangement without pain (TMD_ID, n=370), TMD with joint pain (TMD_J, n=571), TMD with muscle pain (TMD_M, n=541) and TMD with muscle-joint combined pain (TMD_MJ, $\mathrm{n}=376$ ).

Results: The female ratio was particularly high in the group with TMD_MJ ( $p=0.001)$. The patients with muscle pain and both muscle and joint pain had longer symptom duration $(p=0.004)$ and presented significantly higher scores in pain experience $(p<0.001)$, subjective sleep quality $(\mathrm{p}<0.001)$, pain catastrophizing $(\mathrm{p}<0.001)$ and psychological distress $(\mathrm{p}<0.05)$ except for paranoid-ideation than the groups with only joint problems.

Conclusions: The results of this study highlight the importance of multi-dimensional approach that consider pain disability, sleep quality, and psychological functioning in the management of TMD with muscle component. This study would contribute to a better understanding of interaction between heterogeneous TMD and multiple risk factors in order to build tailored treatment based on different phenotypes.

Key Words: Catastrophization; Pain; Phenotype; Psychological distress; Sleep; Temporomandibular disorder

\section{INTRODUCTION}

Pain is an unpleasant sensory and emotional experience. Unlike protective aspect of acute pain, persistent pain interferes with daily life and may cause psychological distress such as anxiety and fear. Most orofacial pain commonly encountered by dentists originates from the teeth and the cause and treatment of toothache are usually clear, so that there is no great difficulty in diagnosis and treatment [1,2]. However, temporomandibular disorders (TMD), known as

Copyright (c) 2020 Korean Academy of Orofacial Pain and Oral Medicine. All rights reserved.

(c) This is an open-access article distributed under the terms of the Creative Commons Attribution Non-Commercial License (http://creativecommons.org/licenses/by-nc/4.0/), which permits unrestricted non-commercial use, distribution, and reproduction in any medium, provided the original work is properly cited. 
the most common non-dental origin of orofacial pain and the second most common musculoskeletal condition [35], presents complex and multidimensional etiology and heterogeneous nature in its symptoms $[5,6]$. This is mainly caused by characteristics of TMD which embraces various painful and non-painful of clinical conditions that involve the masticatory musculature, the temporomandibular joint (TMJ) and associated structures and often results in some difficulties for diagnosis and management of TMD [7].

There are a variety of factors associated with development and persistence of TMD including age, sex, occlusion, trauma, parafunctional habits, orthodontic treatment and etc [5,8-10]. Besides to these systemic and local factors, psychological factors such as anxiety, depression, somatization and catastrophizing have been recently emphasized [11-19]. A relation of sleep and TMD has been also studied [10,14,20-23]. In recent studies, these factors that influence TMD have been analyzed in accordance with TMD subgroups such as muscle disorder and joint disorders $[4,18,19]$. As in the case with other parts of the body, factors such as pain experience, sleep, and psychological distress are known to affect muscle pain rather than joint pain in TMD $[15,24,25]$.

TMD is a mosaic of clinical signs and symptoms that can be regarded as a set of phenotypes that are affected by various factors including pain sensitivity, pain disability, sleep and psychological functioning. Understanding of different phenotypes of TMD is an essential prerequisite of successful management. However, comparative studies on effects of influential factors between TMD subgroups were scarce in a large sample. The aims of this study were to evaluate association of pain experience, sleep quality and psychological distress with different phenotypes of TMD patients.

\section{MATERIALS AND METHODS}

\section{Subjects}

The included subjects in this study were a total of 1,858 TMD patients (1,186 females, 672 males; mean age $=34.9 \pm 15.9$ years; range $10-95$ years) who visited first at the Orofacial Pain Clinic of Dankook University Dental Hospital (Cheonan, Korea) over a period of two years from 2016 to 2017 for the management of TMD. All participants were asked to complete the questionnaires before the diagnosis, and filled them out in the waiting room. The questionnaires included Brief Pain Inventory (BPI); Graded Chronic Pain Scale (GCPS), Pain Catastrophizing Scale (PCS); Symptom Checklist-90-Revision (SCL-90R); Pittsburgh Sleep Quality Index (PSQI).

Exclusion criteria were as follows: (1) Patients who refused to fill out questionnaires or refused to use their survey data for research purposes, (2) Patients who did not fully complete the questionnaire, (3) Patients who couldn't be given a definite diagnosis about their orofacial pain, (4) Patients diagnosed with mental illness by medical history, (5) Patients who are on chemotherapy or have a history of cancer diagnosis, and (6) Patients diagnosed with other neuropathic or central diseases such as stroke, Parkinson disease and etc.

All patients were assessed by experienced orofacial pain specialists who were trained in accordance with the Diagnostic Criteria for Temporomandibular Disorders (DC/ TMD) [3]. Then, all the TMD patients were classified into four groups as follow (Fig. 1) (1) group 1: TMD with internal derangement without pain (TMD_ID, n=370), (2) group 2: TMD with joint pain (TMD_J, $n=571$ ), (3) group 3: TMD with muscle pain (TMD_M, n=541) and (4) group 4: TMD with muscle-joint combined pain (TMD_MJ, $n=376$ ).

The study plan was reviewed and approved by Institutional

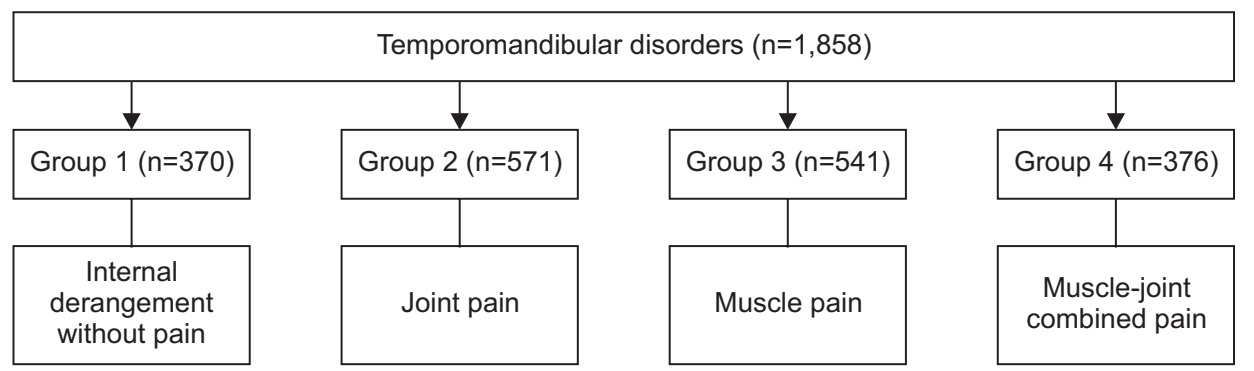

Fig. 1. Flow chart of included patients. 
Review Board (IRB) committee of Dankook University Dental Hospital (IRB no. 2018-03-003) and informed consent was obtained from all the subjects.

\section{Self-Reported Questionnaires}

\section{1) Brief Pain Inventory}

The BPI is a short, simple questionnaire and one of the most widely used measurement tools for assessing clinical pain $[26,27]$. It was presented with $0-10$ scales to assess the pain severity and pain interference. The BPI pain severity is composed the four pain items: 'worst', 'least', 'average' and 'now'. The BPI pain interference includes seven items to measure how much pain has interfered with daily activities, walking, work, mood, enjoyment of life, relations with others, and sleep. The Korean version of BPI was used, and the item related to walking ability was replaced by the question of 'chewing ability' related to orofacial pain [14]. Responses were based on the week before the completion of the BPI.

\section{2) Graded Chronic Pain Scale}

The GCPS assesses the severity of chronic pain and pain disability and consists of seven questions that reflect pain intensity, disability days (loss of work days) due to pain, and interference in daily activities [28]. The pain intensity is calculated as the average of the pain at the time of the interview, the average pain, and the worst pain for the last 6 months (0-100 score). The number of disability days also refers to the days with pain during the past 6 months. GCPS are graded as a 0-IV score, and the meaning of each grade is as follows: (1) 0, no pain, (2) I, low intensity-low disability, (3) II, high intensity-low disability, (4) III, high disabilitymoderately limiting, (5) IV, high disability-severely limiting. Subjects with Grade I and II are classified as low disability group and are considered psychosocially functional with minimal interference or disability associated with their daily living, while subjects with Grade III and IV are classified as high disability group and are considered psychosocially dysfunctional with greater impact on activities of daily living.

\section{3) Pain Catastrophizing Scale}

The PCS developed by Sullivan et al. [29,30] in 1995 is used, and the items relate to the frequency of thoughts and feelings experienced when participants are in pain. It contains 13 items on a 5-point scale with the end points (0) not at all and (4) all the time. The PCS yields a total score and three subscale scores assessing rumination, magnification and helplessness.

\section{4) Symptom Checklist-90-Revision}

SCL-90R has been used to evaluate the respondent's level of psychological well-being using 90 items [31]. This 90-items has a Likert-type scale with 5 point scale (not at all, 0; extremely, 4) for how much each problem had distressed or bothered them during the past 7 days. It evaluates nine symptom dimensions: somatization, obsessive-compulsive, interpersonal sensitivity, depression, anxiety, hostility, phobic anxiety, paranoid ideation, and psychoticism.

\section{5) Pittsburgh Sleep Quality Index}

The PSQI contains 19 self-rated questions which assess a wide variety of factors relating to sleep quality [32]. These 19 items are grouped into seven component scores, which consisted on a scale of 0-3. The seven components are subjective sleep quality, sleep latency, sleep duration, habitual sleep efficiency, sleep disturbances, use of sleeping medications, and daytime dysfunction. These seven component scores were summed to yield a global PSQI score, which ranged from 0 to 21 ; higher scores indicate worse sleep quality.

\section{Statistical Analysis}

One-way ANOVA with Tukey post-hoc analysis was used to analyze the possible influence of sex, pain duration, BPI pain severity and interference, sleep quality, catastrophizing and psychological distress on 4 different phenotypes of TMD. Difference in levels of GCPS disability among 4 TMD groups was compared by Pearson's chi-square test. The significance level for all analyses was set at 0.05. All analyses were done in the PASW Statistics for Windows, Version 18.0 (SPSS Inc., Chicago, IL, USA). 


\section{RESULTS}

\section{Demographic Characteristics of four TMD groups}

A total of 1,858 subjects were included in this study. The demographic data of four groups was shown in the Table 1. The number of patients corresponding to was the highest in the group 2 ( $\mathrm{n}=571)$, and higher in the order of group 3 $(n=541)$, group $4(n=376)$ and group $1(n=370)$. The female ratio was the highest in the group $4(73.4 \%)(p=0.001)$, and there was no sex difference between the other groups. The mean age of group 4 (38.5 years old) was the highest, followed by that of group 2 (36.4 years old), 3 (35.3 years old), and group 1 (28.3 years old) showed the lowest mean age $(\mathrm{p}<0.001)$.

\section{Pain Experience and Sleep Quality of Four TMD Groups}

Pain experience and sleep quality of four different TMD groups was analyzed in the Table 2. Group 1 showed the

Table 1. Demographic data of patients with temporomandibular disorders classified into four groups

\begin{tabular}{|c|c|c|c|c|}
\hline Demographics & Group 1 & Group 2 & Group 3 & Group 4 \\
\hline Patients & $370(19.9)$ & $571(30.7)$ & $541(29.1)$ & $376(20.2)$ \\
\hline Female (\%) & 62.3 & 61.5 & 62.7 & 73.4 \\
\hline Pearson chi-square $\left(\chi^{2}\right)$ & \multicolumn{4}{|c|}{$5.612(p=0.001)$} \\
\hline Age $(y)$ & $28.3 \pm 12.2$ & $36.4 \pm 17.0$ & $35.3 \pm 14.9$ & $38.5 \pm 16.6$ \\
\hline One-way ANOVA (F-value) & \multicolumn{4}{|c|}{$30.858(p<0.001)$} \\
\hline
\end{tabular}

Values are presented as number (\%) or mean \pm standard deviation.

Total number of included patients=1,858. Group 1, 2, 3, and 4 indicate internal derangement without pain, joint pain, muscle pain and musclejoint combined pain, respectively.

A p-values were determined by Pearson chi-square for sex and One-way ANONA for age. Results of Tukey post-hoc analysis: Sex: Group 1, 2, $3<$ Group 4. Age: Group $1<$ Group 2, 3 <Group 4.

Table 2. Clinical data and sleep quality of patients with temporomandibular disorders classified into four groups

\begin{tabular}{lcccccc}
\hline Clinical data and PSQI score & Group 1 & Group 2 & Group 3 & Group 4 & F-value & $p$-value \\
\hline Symptom duration & $23.3 \pm 38.6$ & $14.3 \pm 32.5$ & $18.4 \pm 39.4$ & $18.9 \pm 38.9$ & 4.488 & 0.004 \\
Pain severity & $1.6 \pm 2.0$ & $3.4 \pm 2.3$ & $3.6 \pm 2.4$ & $4.0 \pm 2.1$ & 84.284 & $<0.001$ \\
Pain interference & $1.8 \pm 2.0$ & $3.1 \pm 2.5$ & $3.6 \pm 2.5$ & $4.3 \pm 2.7$ & 73.065 & $<0.001$ \\
PSQI score & $7.4 \pm 2.8$ & $8.1 \pm 3.3$ & $8.4 \pm 3.4$ & $9.1 \pm 3.7$ & 17.974 & $<0.001$ \\
\hline
\end{tabular}

PSQI, Pittsburgh Sleep Quality Index.

Values are presented as mean \pm standard deviation.

Group 1, 2, 3, and 4 indicate internal derangement without pain, joint pain, muscle pain and muscle-joint combined pain, respectively. A p-values were determined by One-way ANONA. Results of Tukey post-hoc analysis: Symptom duration: Group $2<$ Group 3 , $4<$ Group 1. Pain severity: Group 1 <Group 2, 3 <Group 4. Pain interference: Group 1 <Group 2 <Group 3 <Group 4. PSQI score: Group I <Group 2, 3 <Group 4.

Table 3. Comparison of pain disability between four groups with temporomandibular disorder

\begin{tabular}{cccccc}
\hline GCPS grade & Group 1 & Group 2 & Group 3 & Group 4 & $\chi^{2}$ \\
\hline Low disability & & & & 155.2 \\
Grade I & $289(78.1)$ & $268(46.9)$ & $240(44.3)$ & $154(40.9)$ & $<0.001$ \\
Grade II & $31(8.3)$ & $136(23.8)$ & $99(18.2)$ & $73(19.4)$ \\
Total & $320(86.4)$ & $404(70.7)$ & $339(62.5)$ & $227(60.3)$ \\
High disability & & & & \\
Grade III & $32(8.6)$ & $123(21.5)$ & $131(24.2)$ & $95(25.2)$ \\
Grade IV & $18(4.9)$ & $44(7.7)$ & $71(13.1)$ & $54(14.3)$ \\
Total & $50(13.5)$ & $167(29.2)$ & $202(37.3)$ & $149(39.5)$
\end{tabular}

GCPS, Graded Chronic Pain Scale.

Values are presented as number (\%).

Group 1, 2, 3, and 4 indicate internal derangement without pain, joint pain, muscle pain and muscle-joint combined pain, respectively. Low disability indicates the sum of Grade I and II and the sum score of Grade III and IV represents the high disability.

A $p$-value was determined by Pearson chi-square test. 
longest symptom duration (28.3 months), and followed by group 3 (18.4 months) and 4 (18.9 months), and group 2 had the shortest duration of symptom (14.3 months) $(p=0.004)$. Among the four groups, group 4 presented the highest scores of PSQI score as well as pain severity and pain interference while the least scores for group $1(\mathrm{p}<0.001)$. Unlike pain severity and PSQI score, pain interference was higher in the group 3 than in the group 2. Table 3 showed pain disability of four TMD groups. The percentage of high disability group (Grade III and IV) was higher in the order of group 4 (39.5\%), group 3 (37.3\%), group 2 (29.2\%) and group $1(19.6 \%)$ and the percentage of low disability group (Grade I and II) was higher in the opposite order (Table 3, $\mathrm{p}<0.001)$.

\section{Psychological Distress of Four TMD Groups}

Psychological distress including pain catastrophizing scale and SCL-90R was compared in four TMD groups (Table 4). All three dimensions of pain catastrophizing including magnification, rumination and helplessness were the highest in group 4, and followed by group 2 and group 3. Group 1 showed the least pain catastrophizing score $(p<0.001)$. Among the nine subcategories of SCL-90R, all dimensions except paranoid ideation showed group difference.
Somatization scores of group 3 and group 4 was higher than those of group 1 and group 2. Depression was higher in the order of group 3, group 4, group 2 and group 1. Group 3 exhibited the highest score in the obsessive-compulsive, phobic-anxiety, anxiety, interpersonal sensitivity, psychoticism and hostility.

\section{DISCUSSION}

This study investigated the various factors that could affect or be the result of TMD. TMD were classified into 4 subgroups which can be largely divided into muscle pain and joint pain/disorder. Among them, the patients with muscle pain and both muscle and joint pain presented significantly higher scores in pain experience, sleep quality and psychological distress than the groups with only joint problems. The outcomes of this study with large cohort clearly support the concept that masticatory muscle pain is clinically distinct from internal derangement and painful TMJ disorders.

\section{Demographic Characteristics of Four TMD Groups}

The demographic data of TMD patients showed that in all TMD groups (TMD with internal derangement without pain,

Table 4. Pain catastrophizing and psychological distress of patients with temporomandibular disorder classified into four groups

\begin{tabular}{|c|c|c|c|c|c|c|}
\hline Catastrophizing and psychological distress & Group 1 & Group 2 & Group 3 & Group 4 & F-value & p-value \\
\hline \multicolumn{7}{|l|}{ PCS } \\
\hline Magnification & $2.8 \pm 2.6$ & $3.5 \pm 2.8$ & $4.0 \pm 3.0$ & $4.4 \pm 3.1$ & 20.776 & $<0.001$ \\
\hline Rumination & $3.1 \pm 3.9$ & $4.4 \pm 4.3$ & $5.1 \pm 6.4$ & $5.6 \pm 4.7$ & 18.084 & $<0.001$ \\
\hline Helplessness & $4.0 \pm 3.7$ & $5.8 \pm 5.0$ & $6.5 \pm 5.0$ & $7.3 \pm 6.2$ & 30.738 & $<0.001$ \\
\hline \multicolumn{7}{|l|}{ SCL-90R } \\
\hline Somatization & $44.4 \pm 7.1$ & $45.6 \pm 8.4$ & $48.0 \pm 8.9$ & $48.0 \pm 9.1$ & 19.081 & $<0.001$ \\
\hline Obsessive-compulsive & $40.9 \pm 8.5$ & $42.2 \pm 9.4$ & $43.6 \pm 10.1$ & $42.8 \pm 9.9$ & 6.223 & $<0.001$ \\
\hline Interpersonal sensitivity & $40.9 \pm 8.1$ & $41.8 \pm 8.3$ & $43.9 \pm 15.1$ & $42.7 \pm 9.4$ & 6.404 & $<0.001$ \\
\hline Depression & $40.6 \pm 7.7$ & $41.6 \pm 8.7$ & $43.5 \pm 10.2$ & $42.8 \pm 9.8$ & 8.684 & $<0.001$ \\
\hline Anxiety & $41.9 \pm 7.0$ & $43.2 \pm 15.0$ & $45.1 \pm 23.7$ & $43.8 \pm 9.1$ & 3.061 & 0.027 \\
\hline Hostility & $43.3 \pm 6.7$ & $44.0 \pm 7.8$ & $45.1 \pm 7.9$ & $44.9 \pm 8.2$ & 5.185 & 0.001 \\
\hline Phobic-anxiety & $43.8 \pm 6.7$ & $44.2 \pm 7.7$ & $45.4 \pm 8.8$ & $44.9 \pm 8.6$ & 3.365 & 0.018 \\
\hline Paranoid ideation & $41.9 \pm 16.7$ & $41.7 \pm 7.2$ & $42.8 \pm 8.1$ & $43.2 \pm 17.2$ & 1.617 & 0.183 \\
\hline Psychoticism & $41.5 \pm 5.6$ & $42.4 \pm 6.8$ & $43.8 \pm 8.2$ & $42.6 \pm 7.4$ & 8.020 & $<0.001$ \\
\hline
\end{tabular}

PCS, pain catastrophizing scale; SCL-90R, Symptom Check List-90Revised.

Values are presented as mean \pm standard deviation.

Group 1, 2, 3, and 4 indicate internal derangement without pain, joint pain, muscle pain and muscle-joint combined pain, respectively. A p-values were determined by One-way ANONA. Results of Tukey post-hoc analysis: Magnification/Rumination/Helplessness: Group 1<Group 2, 3 <Group 4. Somatization: Group 1, 2 <Group 3, 4. Obsessive-compulsive; Phobic-anxiety; Anxiety: Group 1 <Group 2, 4 <Group 3. Interpersonal sensitivity; Psychoticism: Group 1, 2 <Group 4 <Group 3. Depression: Group 1 <Group 2 <Group 4 <Group 3. Hostility: Group 1 $<$ Group $2<$ Group 3, 4. 
joint pain, muscle pain, muscle-joint combined pain), 60\%$70 \%$ of the subjects were female. This strong female preponderance in TMD population is consistent with the findings of previous studies [11,12,33]. Although there is still no clear consensus for this sex difference, it can be inferred that this strong female representation is affected by behavioral, psychosocial, hormonal and constitutional differences [9]. The effects of the hormone estrogen, as potential contributing factor on the etiology of TMD were most extensively studied in the previous literature $[8,34,35]$. Among the groups, the proportion of female in muscle-joint combined pain group was significantly higher than that in the other groups (Table $1, \mathrm{p}=0.001$ ). This might be attributable to the fact that female tend to present more multiple pain sites than male in chronic pain [36,37].

The mean age of the muscle-joint combined pain group (38.5 years old) was the highest, followed by the mean age of the joint pain group (36.4 years old), the muscle pain group (35.3 years old) and the group of internal derangement without pain (28.3 years old) showed the lowest mean age $(\mathrm{p}<0.001)$. From the previous review on TMJ disorders, internal derangement tends to occur before pain-related TMD [38]. Manfredini et al. [39] reported that when TMD patients were divided into patients with internal derangement (with or without muscle and/or arthralgia) and those with arthritis/arthrosis, there was a significant difference in the mean age of these patients. In addition, Guarda-Nardini et al. [40] suggested that there is a change in the pattern of clinical diagnosis according to the age. They reported that the number of patients with internal derangement decreased with age and the number of patients with arthrosis/ arthritis increased with age [40]. Although categorization of TMD subgroup in both studies are not consistent with that of the current study, their results were comparable with the finding of this study that the mean ages of joint pain and muscle-joint combined pain group were higher than that of internal derangement patients.

\section{Pain Experience and Sleep Quality of Four TMD Groups}

Symptom duration was longer in TMD with internal derangement without pain than the other TMD groups with pain (joint pain, muscle pain, muscle-joint combined pain) (Table $2, \mathrm{p}=0.004$ ). This may be partly explained by the fact that internal derangement patients without pain tend not to visit a hospital because it does not greatly affect the quality of life compared to painful conditions. In addition, muscle pain and muscle-joint combined pain group had significantly longer symptom duration than joint pain group. This result indicates that masticatory muscle pain lasts longer than TMJ pain. Huang et al. [24] reported that the presence of muscle pain (with or without arthralgia) was significantly associated with various risk factors (i.e. clenching, female sex, somatization etc.) but no significant associations were found in the arthralgia-only group. Therefore, multiple risk factors of muscle pain might contribute to the persistence of pain if these factors are not adequately managed.

Group with muscle-joint combined pain showed the highest pain severity and interference, followed by muscle pain group. Not surprisingly, group without pain presented the least pain severity and interference (Table 2, $\mathrm{p}<0.001$ ). This finding is consistent with a previous study comparing pain severity and pain-related interference by subdividing TMD into muscle problem, joint problem, and musclejoint combined problem [19]. The presence of multiple pain sites can be interpreted as an increase and/or spreading of pain. Previous studies reported that the more pain areas, the greater the pain intensity [37,41]. Also, it is not surprising to infer that the more pain at multiple areas, the greater the pain interference in daily jaw function.

Like pain severity and interference, the PSQI score was the highest in the muscle-joint combined pain group among the four groups, while it was the lowest in the group of internal derangement without pain. Up to $77 \%$ of orofacial pain patients report poor sleep quality including reduced quantity and quality of sleep [22]. There are several studies that involve psychological distress such as depression and anxiety and clinical pain experience including pain intensity and pain chronicity in the interaction process of sleep and pain $[22,42,43]$. Particularly, it is reported that anxiety and depression are closely related to sleep quality $[22,43]$. Although the effect of pain intensity on sleep is currently controversial, pain perception alone may directly disrupt sleep [22]. Therefore, poor sleep quality, increased pain and psychological distress can affect each other and this vicious cycle might aggravate pain experience of TMD patients. Based on this fact, it can be inferred that the PSQI score of 
the group of internal derangement without pain is low because the pain intensity and psychological distress are the lowest. In this context, sleep quality should be mainly considered in the management of muscle-joint combined pain group for successful treatment.

On the other hand, pain disability, limitation of the ability to work within normal range due to pain, is associated with important risk factors for pain including depression, somatization, pain duration and widespread pain [44]. In Dworkin's study [45], patients were classified according to their grade of pain disability, regardless of physical diagnosis in order to confirm the importance of pain disability evaluation in the treatment of TMD. In this study, the prognosis of conservative treatment in patients with TMD depends on the disability level rather than physical diagnosis. In the current study, low pain disability (Grade I and II) was observed mainly in group 1 (internal derangement without pain) and group 2 (joint pain) while high disability (Grade III and IV) was mainly found in TMD with muscle pain and TMD with muscle-joint combined pain (Table 3, p<0.001). These findings are consistent with the previous studies that TMD patients with joint disorder and joint pain show lower disability compared with TMD patients with muscle pain $[44,46]$. Therefore, assessment of pain disability level is considered to be an indispensable component in the treatment of TMD patients with muscle pain.

\section{Psychological Distress of Four TMD Groups}

In chronic pain patients, pain catastrophizing, as a precursor of pain problems rather than a consequence, has been reported to play an important role in the chronic pain experience, contribute to pain intensity, pain-related disability and psychological distress [47]. In this study, pain catastrophizing was the highest in the patients with musclejoint combined pain who also presented the highest pain severity and interference (Table $4, \mathrm{p}<0.001$ ). Therefore, it can be interpreted that there is a close relationship between pain catastrophizing and pain severity and interference. Park et al. [18] suggested that pain catastrophizing of TMD patients had a positive correlation with pain severity and pain interference which is consistent with the findings of this study.

Recent studies divided TMD into two groups of muscle pain and joint pain to assess their association with psychological distress [24,48-51]. However, they focused on the particular dimension including somatization, depression and anxiety [24,48-51]. In addition, a study of 96 TMD patients reported that the mean scores of the SCL-90R were higher in the group with muscle pain than in the group with joint pain but failed to reach statistically significant difference [51]. This statistical result might be attributable to the small sample size of the study. The current study has the strength that all dimensions of psychological distress using the SCL-90R were investigated in a large sample of 1,858 TMD patients. According to this study, psychological distress (Somatization, Obsessive-Compulsive, Interpersonal sensitivity, Depression, Anxiety, Hostility, Phobic-anxiety, Paranoid ideation, Psychoticism) was generally lower in TMD with internal derangement without pain and higher in TMD with muscle pain, and all of the dimensions except the paranoid ideation were statistically significant $(\mathrm{p}<0.05)$. This study highly suggests that psychological distress is a prominent risk factor for TMD patients with muscle component.

In conclusion, in this cross-sectional study, quantitative and qualitative gradient of pain experience, sleep quality and psychological distress in patients with different phenotypes of temporomandibular disorders was clearly seen. Among TMD patients, patients with internal derangement without pain showed the least pain profile. In painful TMD, muscle pain rather than joint pain was significantly associated with pain experience, sleep quality, and psychological distress. The more pain sites, the higher pain experience and psychological distress, the lower the sleep quality.

The results of this study highlight the importance of multi-dimensional approach that consider pain disability, sleep quality, and psychological functioning in the management of TMD with muscle component. This study would contribute to a better understanding of interaction between heterogeneous TMD and multiple risk factors in order to build tailored treatment based on different phenotypes.

\section{CONFLICT OF INTEREST}

No potential conflict of interest relevant to this article was reported. 


\section{ORCID}

\author{
Hee Hun Choi \\ https://orcid.org/0000-0002-8224-8231 \\ Hye-Kyoung Kim \\ https://orcid.org/0000-0002-0734-5533 \\ Mee-Eun Kim \\ https://orcid.org/0000-0001-9332-532X
}

\section{REFERENCES}

1. Traebert J, de Lacerda JT, Fischer TK, Jinbo Y. Dental caries and orofacial pain trends in 12-year-old school children between 1997 and 2003. Oral Health Prev Dent 2005;3:243-248.

2. Scully C. Oral and maxillofacial medicine: the basis of diagnosis and treatment. 2nd ed. Edinburgh: Churchill Livingstone; 2008. pp. 98-108.

3. Schiffman E, Ohrbach R, Truelove E, et al. Diagnostic Criteria for Temporomandibular Disorders (DC/TMD) for clinical and research applications: recommendations of the International RDC/TMD Consortium Network and Orofacial Pain Special Interest Group. J Oral Facial Pain Headache 2014;28:6-27.

4. Rollman GB, Gillespie JM. The role of psychosocial factors in temporomandibular disorders. Curr Rev Pain 2000;4:71-81.

5. Manfredini D, Guarda-Nardini L, Winocur E, Piccotti F, Ahlberg J, Lobbezoo F. Research diagnostic criteria for temporomandibular disorders: a systematic review of axis I epidemiologic findings. Oral Surg Oral Med Oral Pathol Oral Radiol Endod 2011;112:453462.

6. Romero-Reyes M, Uyanik JM. Orofacial pain management: current perspectives. J Pain Res 2014;7:99-115.

7. Okeson JP. The classification of orofacial pains. Oral Maxillofac Surg Clin North Am 2008;20:133-144, v.

8. Poveda Roda R, Bagan JV, Díaz Fernández JM, Hernández Bazán S, Jiménez Soriano Y. Review of temporomandibular joint pathology. Part I: classification, epidemiology and risk factors. Med Oral Patol Oral Cir Bucal 2007;12:E292-E298.

9. Oral K, Bal Küçük B, Ebeoğlu B, Dinçer S. Etiology of temporomandibular disorder pain. Agri 2009;21:89-94.

10. Rossetti LM, Pereira de Araujo Cdos R, Rossetti PH, Conti PC. Association between rhythmic masticatory muscle activity during sleep and masticatory myofascial pain: a polysomnographic study. J Orofac Pain 2008;22:190-200.

11. LeResche L. Epidemiology of temporomandibular disorders: implications for the investigation of etiologic factors. Crit Rev Oral Biol Med 1997;8:291-305.

12. Pereira LJ, Pereira-Cenci T, Pereira SM, et al. Psychological factors and the incidence of temporomandibular disorders in early adolescence. Braz Oral Res 2009;23:155-160.

13. Guarda-Nardini L, Pavan C, Arveda N, Ferronato G, Manfredini D. Psychometric features of temporomandibular disorders patients in relation to pain diffusion, location, intensity and duration. J
Oral Rehabil 2012;39:737-743.

14. Song KW, Kim ME. Sleep quality of patients with temporomandibular disorders: relationship to clinical and psychological characteristics. J Oral Med Pain 2015;40:155-162.

15. Jaspers JP, Heuvel F, Stegenga B, de Bont LG. Strategies for coping with pain and psychological distress associated with temporomandibular joint osteoarthrosis and internal derangement. Clin J Pain 1993;9:94-103.

16. Ferrando M, Andreu Y, Galdón MJ, Durá E, Poveda R, Bagán JV. Psychological variables and temporomandibular disorders: distress, coping, and personality. Oral Surg Oral Med Oral Pathol Oral Radiol Endod 2004;98:153-160.

17. McCreary CP, Clark GT, Merril RL, Flack V, Oakley ME. Psychological distress and diagnostic subgroups of temporomandibular disorder patients. Pain 1991;44:29-34.

18. Park JH, Kim HK, Kim KS, Kim ME. Pain catastrophizing for patients with temporomandibular disorders. J Oral Med Pain 2015;40:47-54.

19. Choi SH, Kim KS, Kim ME. Pain disability of orofacial pain patients. J Oral Med Pain 2009;34:217-225.

20. Schmitter M, Kares-Vrincianu A, Kares H, Bermejo JL, Schindler HJ. Sleep-associated aspects of myofascial pain in the orofacial area among temporomandibular disorder patients and controls. Sleep Med 2015;16:1056-1061.

21. Kim SA, Yang KI, Oh KY, Hwangbo Y. Association between sleep quality and myofascial pain syndrome in Korean adults: questionnaire based study. J Musculoskelet Pain 2014;22:232-236.

22. Riley JL 3rd, Benson MB, Gremillion HA, et al. Sleep disturbance in orofacial pain patients: pain-related or emotional distress? Cranio 2001;19:106-113.

23. Yatani H, Studts J, Cordova M, Carlson CR, Okeson JP. Comparison of sleep quality and clinical and psychologic characteristics in patients with temporomandibular disorders. J Orofac Pain 2002;16:221-228.

24. Huang GJ, LeResche L, Critchlow CW, Martin MD, Drangsholt MT. Risk factors for diagnostic subgroups of painful temporomandibular disorders (TMD). J Dent Res 2002;81:284-288.

25. Jung JS, Hur YK, Choi JK. Evaluation of quality of life in patient with temporomandibular disorders. J Oral Med Pain 2006;31:127139.

26. Cleeland CS, Ryan KM. Pain assessment: global use of the Brief Pain Inventory. Ann Acad Med Singap 1994;23:129-138.

27. Radbruch L, Loick G, Kiencke P, et al. Validation of the German version of the Brief Pain Inventory. J Pain Symptom Manage 1999;18:180-187.

28. Von Korff M, Ormel J, Keefe FJ, Dworkin SF. Grading the severity of chronic pain. Pain 1992;50:133-149.

29. Sullivan MJL. The Pain Catastrophizing Scale [Internet]. Montreal: Frederic Chappe; c2019 [cited 2020 Aug 18]. Available form: http://sullivan-painresearch.mcgill.ca/pcs.php

30. Sullivan MJL, Bishop SR, Pivik J. The Pain Catastrophizing Scale: development and validation. Psychol Assess 1995;7:524-532.

31. Derogatis LR. SCL-90 R: administration, scoring and procedures. Baltimore: Clinical Psychometric Research; 1977.

32. Buysse DJ, Reynolds CF 3rd, Monk TH, Berman SR, Kupfer DJ. 
The Pittsburgh Sleep Quality Index: a new instrument for psychiatric practice and research. Psychiatry Res 1989;28:193-213.

33. Dworkin SF, LeResche L, Von Korff MR. Diagnostic studies of temporomandibular disorders: challenges from an epidemiologic perspective. Anesth Prog 1990;37:147-154.

34. LeResche L, Saunders K, Von Korff MR, Barlow W, Dworkin SF. Use of exogenous hormones and risk of temporomandibular disorder pain. Pain 1997;69:153-160.

35. Dao TT, Knight K, Ton-That V. Modulation of myofascial pain by the reproductive hormones: a preliminary report. J Prosthet Dent 1998;79:663-670.

36. Andersson HI, Ejlertsson G, Leden I, Rosenberg C. Chronic pain in a geographically defined general population: studies of differences in age, gender, social class, and pain localization. Clin J Pain 1993;9:174-182.

37. Perquin CW, Hazebroek-Kampschreur AA, Hunfeld JA, et al. Pain in children and adolescents: a common experience. Pain 2000;87:51-58.

38. Tatli U, Machon V. Internal derangements of the temporomandibular joint: diagnosis and management. In: Emes Y, ed. Temporomandibular joint pathology-current approaches and understanding. Intech Open; 2018. pp. 436-476.

39. Manfredini D, Piccotti F, Ferronato G, Guarda-Nardini L. Age peaks of different RDC/TMD diagnoses in a patient population. $\mathrm{J}$ Dent 2010;38:392-399.

40. Guarda-Nardini L, Piccotti F, Mogno G, Favero L, Manfredini D. Age-related differences in temporomandibular disorder diagnoses. Cranio 2012;30:103-109.

41. Hadjimichael 0, Kerns RD, Rizzo MA, Cutter G, Vollmer T. Persistent pain and uncomfortable sensations in persons with multiple sclerosis. Pain 2007;127:35-41.

42. Lavigne GJ, Nashed A, Manzini C, Carra MC. Does sleep differ among patients with common musculoskeletal pain disorders? Curr Rheumatol Rep 2011;13:535-542.

43. Sayar K, Arikan M, Yontem T. Sleep quality in chronic pain patients. Can J Psychiatry 2002;47:844-848.

44. Arnow BA, Blasey CM, Constantino MJ, et al. Catastrophizing, depression and pain-related disability. Gen Hosp Psychiatry 2011;33:150-156.

45. Dworkin SF, Turner JA, Mancl L, et al. A randomized clinical trial of a tailored comprehensive care treatment program for temporomandibular disorders. J Orofac Pain 2002;16:259-276.

46. Manfredini D, Ahlberg J, Winocur E, Guarda-Nardini L, Lobbezoo F. Correlation of RDC/TMD axis I diagnoses and axis II pain-related disability. A multicenter study. Clin Oral Investig 2002;15:749-756.

47. Severeijns R, Vlaeyen JW, van den Hout MA, Weber WE. Pain catastrophizing predicts pain intensity, disability, and psychological distress independent of the level of physical impairment. Clin J Pain 2001;17:165-172.

48. Yap AU, Dworkin SF, Chua EK, List T, Tan KB, Tan HH. Prevalence of temporomandibular disorder subtypes, psychologic distress, and psychosocial dysfunction in Asian patients. J Orofac Pain 2003;17:21-28.

49. Yap AU, Tan KB, Chua EK, Tan HH. Depression and somatization in patients with temporomandibular disorders. J Prosthet Dent 2002;88:479-484.

50. Celić R, Braut V, Petricević N. Influence of depression and somatization on acute and chronic orofacial pain in patients with single or multiple TMD diagnoses. Coll Antropol 2011;35:709-713.

51. Manfredini D, Marini M, Pavan C, Pavan L, Guarda-Nardini L. Psychosocial profiles of painful TMD patients. J Oral Rehabil 2009;36:193-198. 